We plan to use our findings to educate local GPs about the risk factors that should trigger early palliative care referral. A tool to aid the early recognition of risk is currently in development and will be integrated into Pilgrims Hospice referral forms in the future.

\section{P-92 IMPROVING TIMELY ACCESS TO SPECIALIST PALLIATIVE CARE, USING QUALITY IMPROVEMENT (QI) METHODOLOGY}

Sian Burgess, Lisa Corbett, Emma Barclay. St Ann's Hospice, UK

\subsection{6/bmjspcare-2016-001245.115}

The aim of the project is to enable more people who are triaged as appropriate for admission by the multi-disciplinary team, to be admitted and receive timely specialist in-patient palliative care.

Background

The rationale behind the project was that:

- Data indicated an increasing demand on specialist palliative care beds

- There was evidence of the impact of delayed discharges on achieving timely access

- There is need to educate society about the changing role of specialist palliative care.

Aim The aim is to increase the number of appropriate admissions from $70 \%$ to $75 \%$.

Method The project uses Quality Improvement methodology as the mechanism for improving practice. The driver diagram below demonstrates how we structured our project.

Small change ideas are being used to slowly make improvements that are effective and sustainable. An example of one change was to review the referral form and admission documentation to ensure from the point of referral that patients understand the reason for their in-patient hospice care and the potential for discharge.

The project is based on the Model for Improvement tool.

Results We are using a measurement strategy to map and evaluate our progress. We are making significant progress as for the last eight months we have surpassed our original target and reached $79 \%$. There are further change ideas that we intend to explore to help with sustainability and spread. One of these is holding a round table discussion with external partners to look at ways they can support the discharge process.

Opportunities We are intending that this project will enable us to maximise available resources whilst at the same time improve access to specialist palliative care to more people in a more timely way.

\section{P-93 REACHING OUT - IMPROVING CARE BEYOND OUR HOSPICE WALLS}

Kathryn Hamling, Kenny Steele. Highland Hospice, Inverness, UK

\subsection{6/bmjspcare-2016-001245.116}

Highland Hospice has a proud history of delivering and promoting high quality palliative care for our community, geographically the size of Belgium with a population of just over 220000 .

The sphere of influence of the hospice is far greater than the direct care we provide to patients, families and carers. It is acknowledged that while direct care can (and does) provide the highest quality response for the individual it is resource intensive. Indirect support can however potentially support many more people especially those who live in the more remote areas of our wide geographical area. As a result we are currently piloting a number of innovative and creative responses to deal with these geographic and demographic challenges using digital communication. In this way we are supporting more people to a higher standard at home or in a care home environment, increasing our value to the wider health and social care system and providing better quality care for those in need.

This outreaching of care is being developed in a number of different ways:-

- Using ECHO Highland Hospice Scotland - a collaborative web-based model of education and care management that moves knowledge without moving people.

- HHeLP - Highland Hospice electronically Linking People an interactive, secure internet portal developed to provide

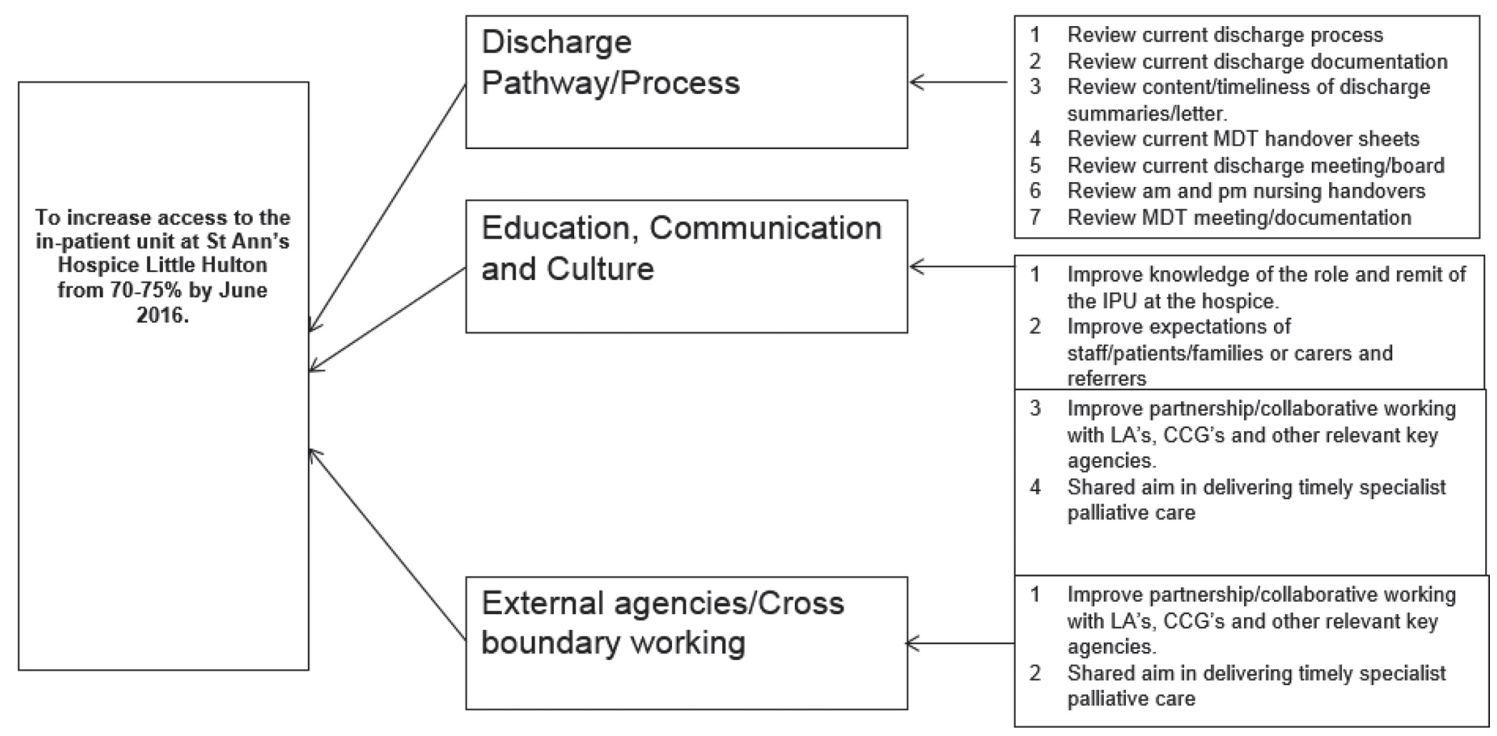

Abs P-92 Table 1 